\title{
Multilevel Comparisons of Hospital Discharge among Older Adults with a Fall-Related Hospitalization
}

\author{
Samuel D. Towne Jr., (D), Kayla Fair, Matthew Lee Smith, \\ Diane M. Dowdy, SangNam Ahn, Obioma Nwaiwu, and \\ Marcia G. Ory
}

Objective. We examined multilevel factors associated with hospital discharge status among older adults suffering a fall-related hospitalization.

Data Sources. The 2011-2013 ( $n=131,978)$ Texas Inpatient Hospital Discharge Public-Use File was used.

Study Design/Methods. Multilevel logistic regression analyses estimated the likelihood of being discharged to institutional settings versus home.

Principal Findings. Factors associated with a greater likelihood of being discharged to institutional settings versus home/self-care included being female, white, older, having greater risk of mortality, receiving care in a non-teaching hospital, having Medicare (versus Private) coverage, and being admitted from a non-health care facility (versus clinical referral).

Conclusions. Understanding risk factors for costly discharges to institutional settings enables targeted fall-prevention interventions with identification of at-risk groups and allows for identifying policy-related factors associated with discharge status.

Key Words. Falls, hospital discharge, older adults, Medicare

Falls disproportionately affect older adults and when falls do occur, they result in serious injury at a much greater rate than among younger individuals (Sterling, O'Connor, and Bonadies 2001), thereby disproportionately affecting the already hard-hit U.S. health care delivery and finance system. Those with a previous history of a fall are more likely to enter costly institutionalized settings, namely nursing homes (Tinetti and Williams 1997). Identifying factors associated with discharge location can help key stakeholders be better informed to identify the best solutions to prevent falls or a recurrent fall(s).

Some have suggested that focusing on post-acute discharge may hold major implications for reducing overall spending (Das et al. 2016; Mor, 
Rahman, and McHugh 2016). Focusing on discharging patients to less costly locations (e.g., home) has been suggested as a major factor in this process of lowering hospital spending (Mor, Rahman, and McHugh 2016). Large variation in the price of medical care may be attributed to variation among hospitals based on various measures such as performance (Das et al. 2016). Thus, accounting for hospital-level variation (e.g., using multilevel analyses) is critical (Rice and Leyland 1996). Ongoing surveillance of fall-related hospitalization is needed to ensure the most current evidence is available to inform policy.

\section{Aims}

Although multiple studies have examined injuries related to serious falls (Masud and Morris 2001; Smith et al. 2010), few studies have investigated factors that may contribute to the discharge location of a patient following a traumatic fall (Lim, Hoffmann, and Brasel 2007). Therefore, our objective was to examine multilevel factors associated with hospital discharge status among older adults suffering a fall-related hospitalization. We aimed to examine fallrelated hospitalization by (1) overall distribution, (2) discharge location, and (3) to identify multilevel factors associated with discharge to institutional settings versus to home or self-care (routine discharge)-henceforth HSC or home health care (HHC). Multiple definitions of discharge to home were included given discharge to home with self-care may incur different costs than being discharged to home with the inclusion of HHC.

Address correspondence to Samuel D. Towne Jr., Ph.D., M.P.H., C.P.H., Department of Health Promotion \& Community Health Sciences, School of Public Health, Texas A\&M University, 1266 TAMU, College Station, TX; e-mail: towne@sph.tamhsc.edu. Kayla Fair, Dr.P.H., is with the Center for Depression Research and Clinical Care, Department of Psychiatry, University of Texas Southwestern Medical Center, Dallas, TX. Matthew Lee Smith, Ph.D., M.P.H., C.H.E.S., is with the Department of Health Promotion and Behavior, College of Public Health, The University of Georgia, Athens, GA, and the Department of Health Promotion \& Community Health Sciences, School of Public Health, Texas A\&M University, College Station, TX. Diane M. Dowdy, Ph.D., M.S., B.S.N., is with the Department of Health Promotion \& Community Health Sciences, School of Public Health, Texas A\&M University, College Station, TX. SangNam Ahn, Ph.D., M.P.S.A., is with the Division of Health Systems Management and Policy, School of Public Health, University of Memphis, Memphis, TN, and the Department of Health Promotion \& Community Health Sciences, School of Public Health, Texas A\&M University, College Station, TX. Obioma Nwaiwu, Ph.D., M.D., is with the Department of Family and Preventive Medicine, University of Arkansas for Medical Sciences, Little Rock, AR. Marcia G. Ory, Ph.D., M.P.H., is with the Center for Population Health and Aging, Texas A\&M University, School of Public Health, College Station, TX. 


\section{DESIGN AND METHODS}

\section{Data}

The Texas Hospital Inpatient Discharge Public-Use Data for 2011 (base-file $n=2,937,634), 2012$ (base-file $n=2,965,961$ ), and 2013 (base-file $n=2,910,853)$ were used (THHS 2011).

\section{Patient Population}

The target population included older adults (age 65+), suffering a fall-related hospitalization, and being admitted from a non-health care facility (NHCF; $n=40,342,42,864,44,977 ; 2011-2013$, respectively) or a clinical referral ( $n=3,200,3,004,3,221 ; 2011-2013$, respectively). Excluding transfers from another facility (e.g., transfer from a hospital or other health care facility) allowed us to identify those most likely to come from a residential setting (e.g., those aging-in-place) versus institutional settings. These excluded observations represented 11.8 percent of fall-related hospitalizations. Discharges with a primary payment source of "other non-federal programs" $(<0.5$ percent for each year) were excluded due to the inability to identify specific sources of payment. The analytical group included 41,933, 43,989, and 46,056 in 2011, 2012, and 2013, respectively.

\section{Main Outcome Measures}

Discharge location was the dependent variable. Being discharged home without any additional care (self-care) versus being discharged home with additional care (e.g., HHC) is associated with different medical costs. Thus, we separated discharge to one's home into three categories in separate analyses. Category A: All discharges to HSC, that is, home (i.e., self-care/routine discharge), including formal service (i.e., HHC or intravenous therapy [IV]); Category B: Discharges to home without formal service (i.e., HHC or IV); Category C: Discharges to home with formal service (i.e., HHC or IV). Category $\mathrm{A}=\mathrm{B}+\mathrm{C}$ : All other discharges included discharges/transfers to other short-term general hospital; skilled nursing facility; intermediate care facility; cancer center; admitted as inpatient to this hospital; still patient; federal health care facility; hospice-medical facility; within this institution to Medicareapproved swing bed; inpatient rehabilitation facility; Medicare-certified longterm care hospital; Medicaid-certified nursing facility; psychiatric hospital or 
psychiatric distinct part of a hospital; critical access hospital; other outpatient service; and institution outpatient.

Individuals were coded as expired/deceased; discharged to hospicehome; and left against medical advice, where excluded from analyses. Our adjusted model assessed fall-related hospitalizations among those either coming from a non-institutional setting or by way of clinical referrals (CR).

\section{Covariates}

Individual-level factors included in the adjusted model were as follows: sex (male/female); race/ethnicity (non-Hispanic American Indian/Alaska Native, non-Hispanic Asian or Pacific Islander, non-Hispanic black, nonHispanic white, and Hispanic); age group (65-74, 75-84, and 85+); payment source identified as the expected primary source of payment (Title-V or other federal program; Veteran Administration plan or Civilian Health and Medical Program of the Uniformed Services, Medicare; Medicaid; charity, indigent or unknown; workers compensation; other forms of payment (nonfederal/non-charity/non-workers compensation) operationally defined as "private" in the current analyses (liability medical, liability, health maintenance organization, disability insurance, commercial insurance, Blue Cross Blue Shield, automobile medical, indemnity insurance, exclusive provider organization, point of service, preferred provider organization, and central certification); and risk of mortality (minor, moderate, major, or extreme risk upon admission).

Hospital- and community-level factors were also included to account for differences at the hospital-level and individual residence. Hospital-level teaching status was included to account for differences in being a teaching-affiliated facility versus a non-teaching facility. Neighborhood-level characteristics including classification as a large central metropolitan, large fringe metropolitan, medium metropolitan, small metropolitan, micropolitan, and noncore area were included to account for differences associated with rural versus urban residence using the National Center for Health Statistics Urban-Rural Classification Scheme. Source of admission, coded as clinical referral (e.g., the patient was referred by a provider from an outpatient clinic including a physician at the hospital) versus NHCF (e.g., patient residing at home prior to admission), was also included to assess potential differences in patient discharge location/status. To further describe our patient population (descriptive statistics only), we identified ICD-9 codes for the primary diagnosis upon admission and average length of stay (LOS). 


\section{Statistical Analyses}

Analyses were conducted using SAS 9.4 using random coefficient models (RCMs) to assess the likelihood of our outcomes. In adjusted analyses, the payment source associated with the hospital discharge was collapsed into Medicare accounting for over 90 percent of all discharges, Private which accounted for between 5 and 7 percent of all cases, and Other given the relatively small analytical group size among other payers. Intraclass correlation coefficients (ICCs) were calculated using a model without predictors (Bell, Ene, and Schoeneberger 2013). The decision to use RCMs was made based on two factors: (1) the nested nature of the data where individuals are nested within hospitals fits with the theoretical framework of multilevel modeling; (2) the ICCs, while all $<10$ percent, did range approximately $4-9$ percent, indicating some variation in our outcomes was likely attributable to differences among hospitals.

\section{Ethical Approval of Studies and Informed Consent}

Ethical approval was granted through the Texas A\&M University Institutional Review Board (IRB).

\section{RESULTS}

Table 1 presents the distribution of older adults admitted to the hospital for a fall-related injury through non-institutionalized settings (NIS) or CR (referred to collectively henceforth as NIS/CR) prior to admission by selected characteristics. Overall, the number of older adults suffering fall-related hospitalizations who were admitted through NIS/CR prior to admission increased from 41,933 in 2011 , to 43,989 in 2012 , and then to 46,056 in 2013 . When testing for significant differences in our binary outcomes of discharge location (i.e., 1 model for each category A, B, C), we find no evidence to suggest any significant difference $(\alpha=0.01)$ by year. To further describe our patient population, we identified ICD-9 codes for the primary diagnosis. When combining all 3 years of data, we find approximately a third (36 percent) of fall-related hospitalizations were associated with a fracture, while most others were associated with infections (approximately 40 percent) and other diagnosis (e.g., circulatory or respiratory issues). The average LOS was approximately 5 days (range $1-368)$. When stratifying LOS by discharge location, we find the average LOS was 5.8 days for those discharged to an institutional setting versus 4.1 days 


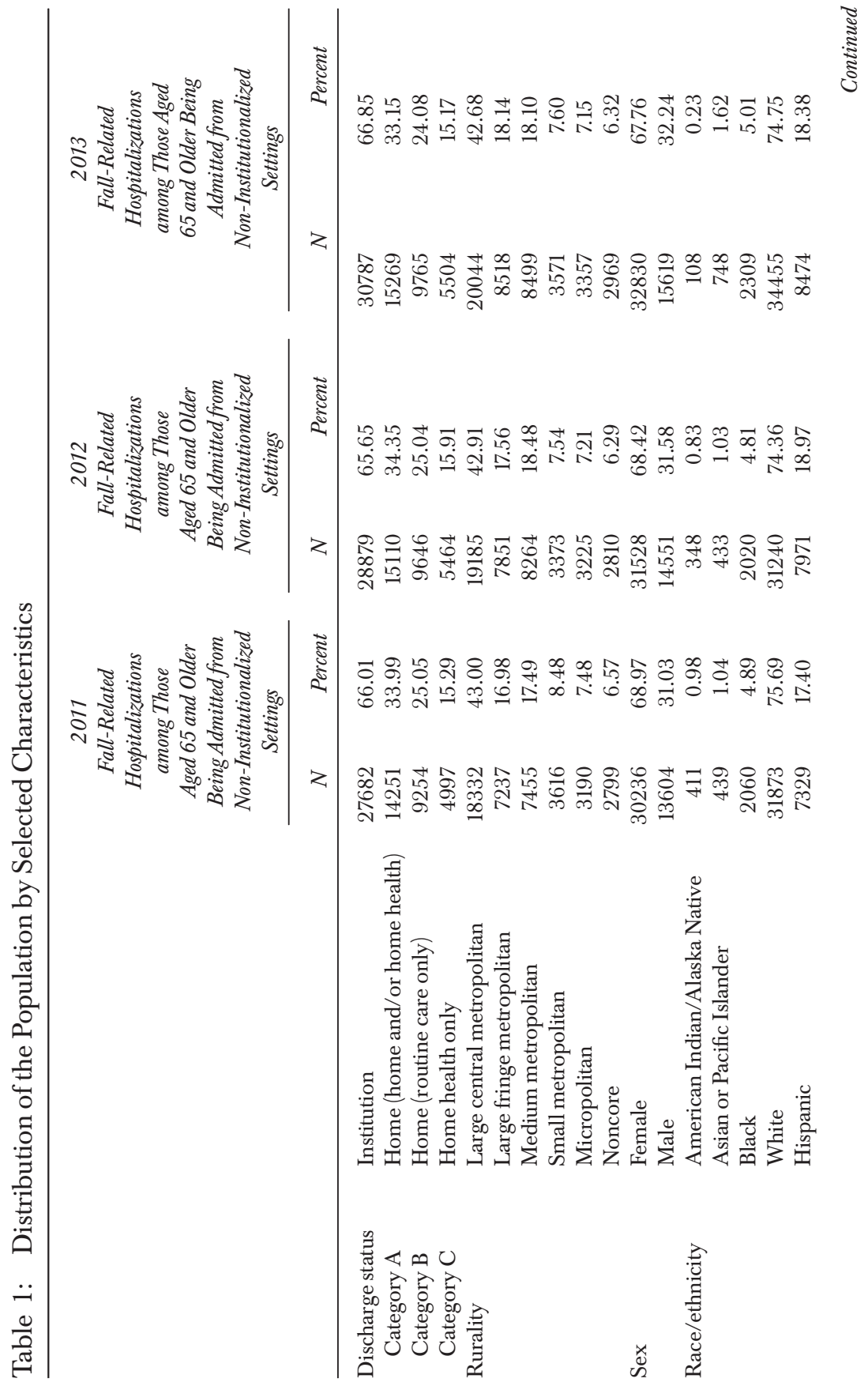




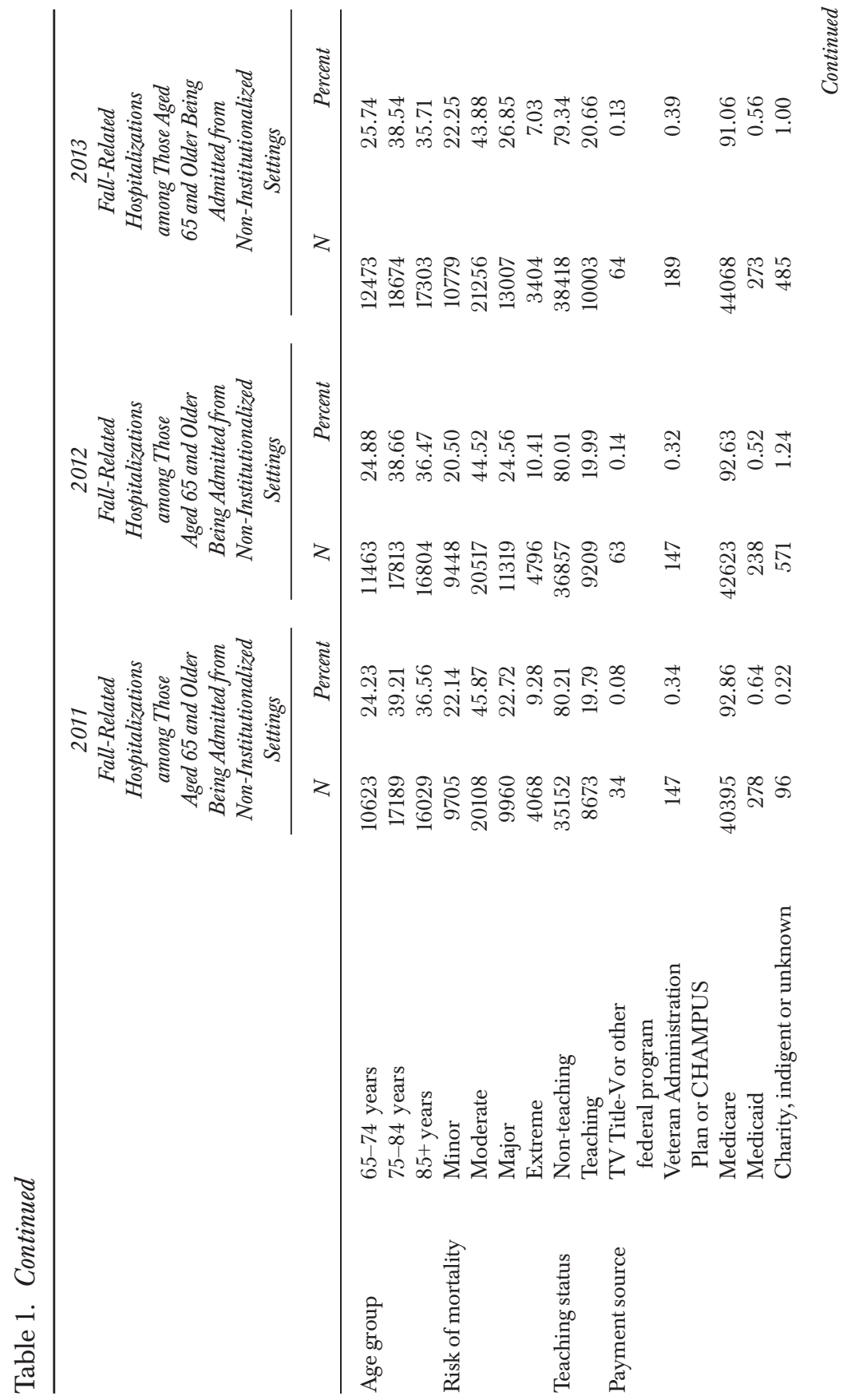




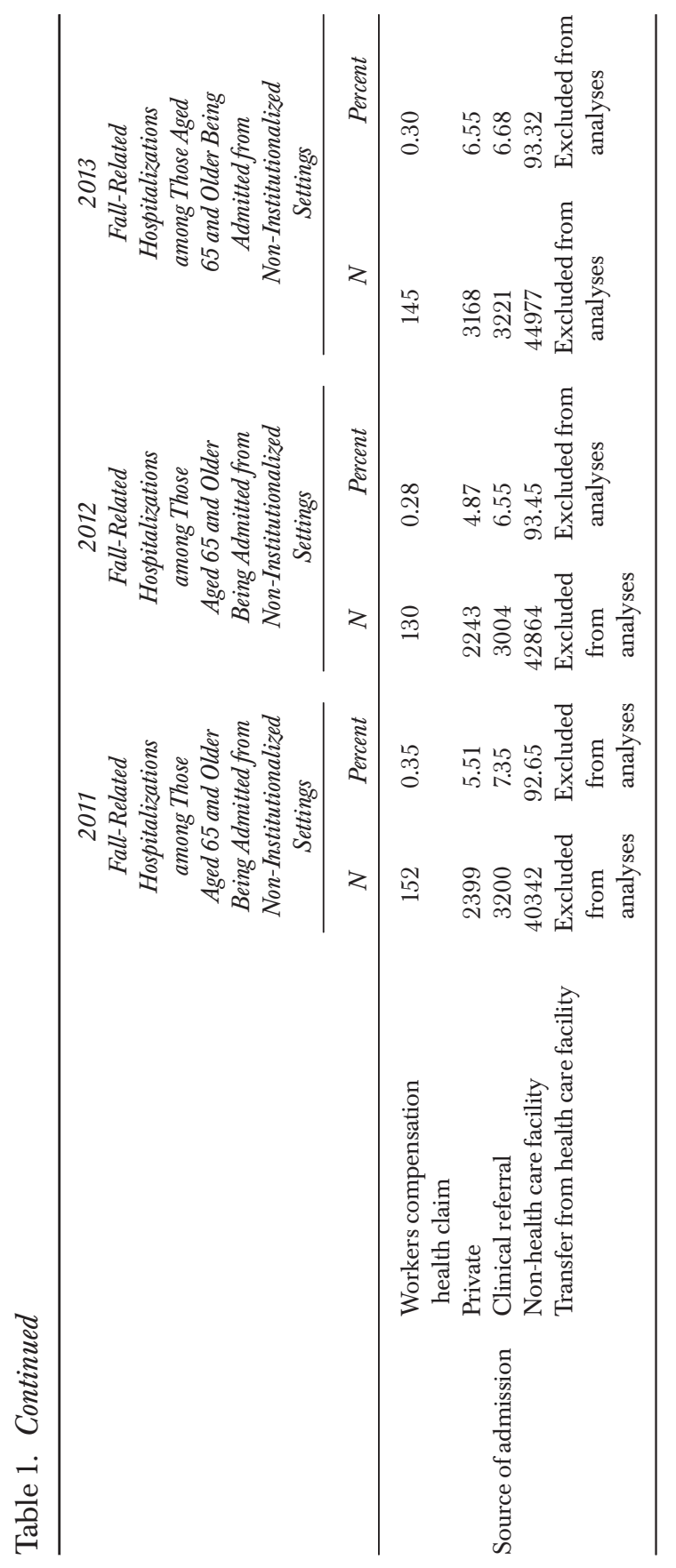


(Category A), 3.7 days (Category B), and 4.9 days (Category C) for being discharged home, respectively.

The proportion of older adults admitted for fall-related injury through NIS/CR that were discharged to $\mathrm{HSC}$ or $\mathrm{HHC}$ was approximately 34 percent in 2011 and 2012, and 33 percent in 2013. The bulk of older adults suffering a fall-related hospitalization admitted through NIS/CR prior to admission were in metropolitan areas. In addition, females accounted for the greatest proportion of fall-related hospitalization, with 69 percent in 2011 and approximately 68 percent in 2012 and 2013. While the majority of older adults suffering a fallrelated hospitalization who were admitted through NIS/CR prior to admission were identified as white, nearly one in five were Hispanic individuals. Individuals aged 65-74 represented nearly one in four discharges among older adults suffering a fall-related hospitalization who were admitted through NIS/CR.

Nearly half of older adults suffering a fall-related hospitalization who were admitted through NIS/CR prior to admission were admitted with a moderate risk of mortality.

For 2011, 2012, and 2013, approximately 79-80 percent of older adults suffering a fall-related hospitalization who were admitted through NIS/CR prior to admission were treated in non-teaching institutions. Medicare represented over 91 percent of all payments sources for older adults suffering a fallrelated hospitalization who were admitted through NIS/CR. Ninety-three percent of the patients in our study were admitted from the home with 7 percent being referred from the clinic.

Table 2 presents the distribution of older adults suffering a fall-related hospitalization who were admitted through NIS/CR prior to admission. The largest segment of all discharges among older adults suffering a fall-related hospitalization who were admitted through NIS/CR prior to admission was represented by discharge to skilled nursing care (approximately 35-37 percent).

\section{Adjusted Analyses}

Discharged to HSC or HHC. Table 3 presents results for the likelihood of being discharged to an institutional health care setting versus $\mathrm{HSC}$ or HHC. Factors associated with $(\alpha=0.05)$ a greater likelihood of being discharged to institutionalized settings among older adults suffering a fall-related hospitalization who were admitted through NIS/CR included the following: being female; being in older age groups versus those aged 65-74; having a higher risk of mortality versus minor risk; being treated in a non-teaching facility versus a teaching facility; and having Medicare as the primary source of payment, after 


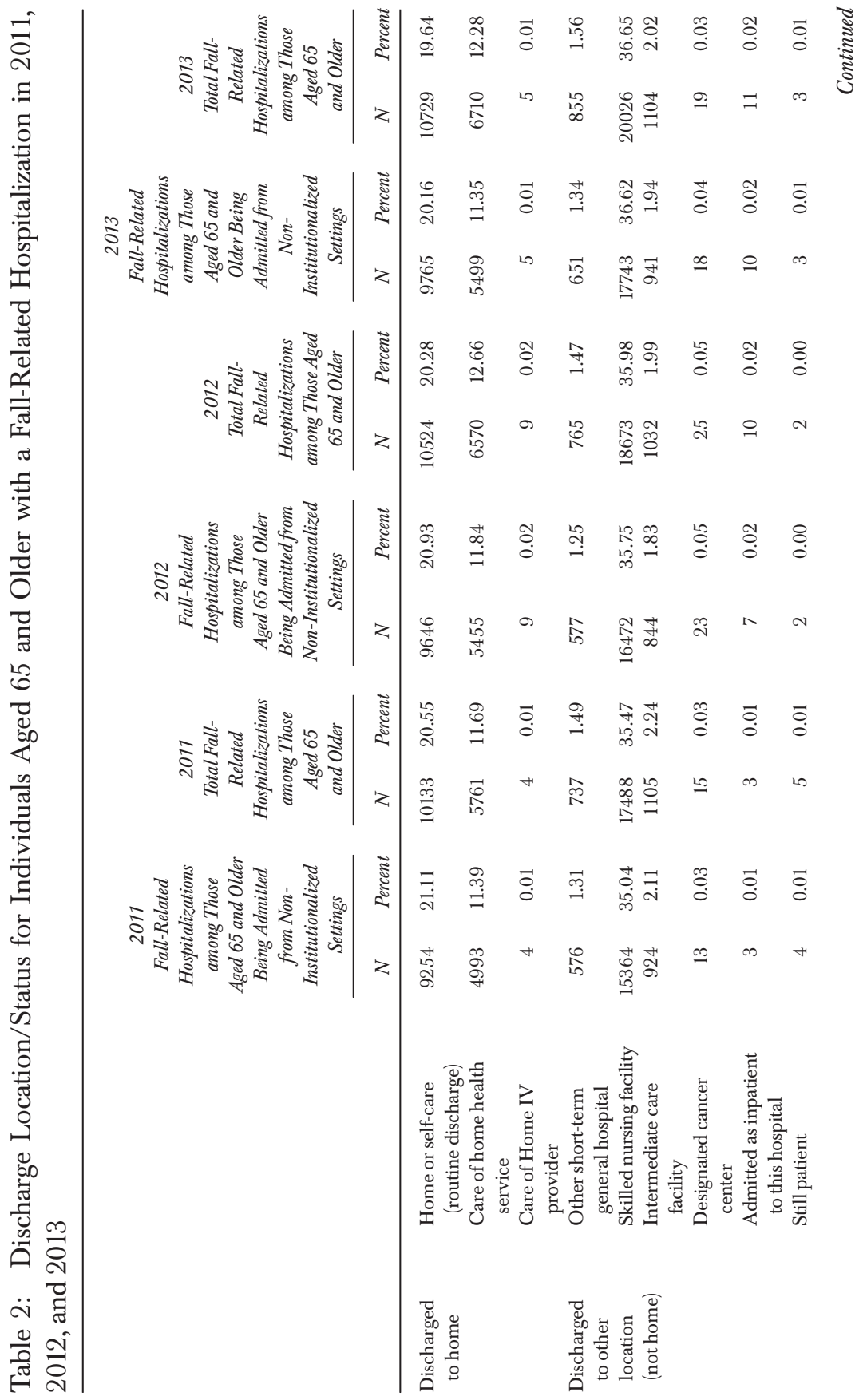




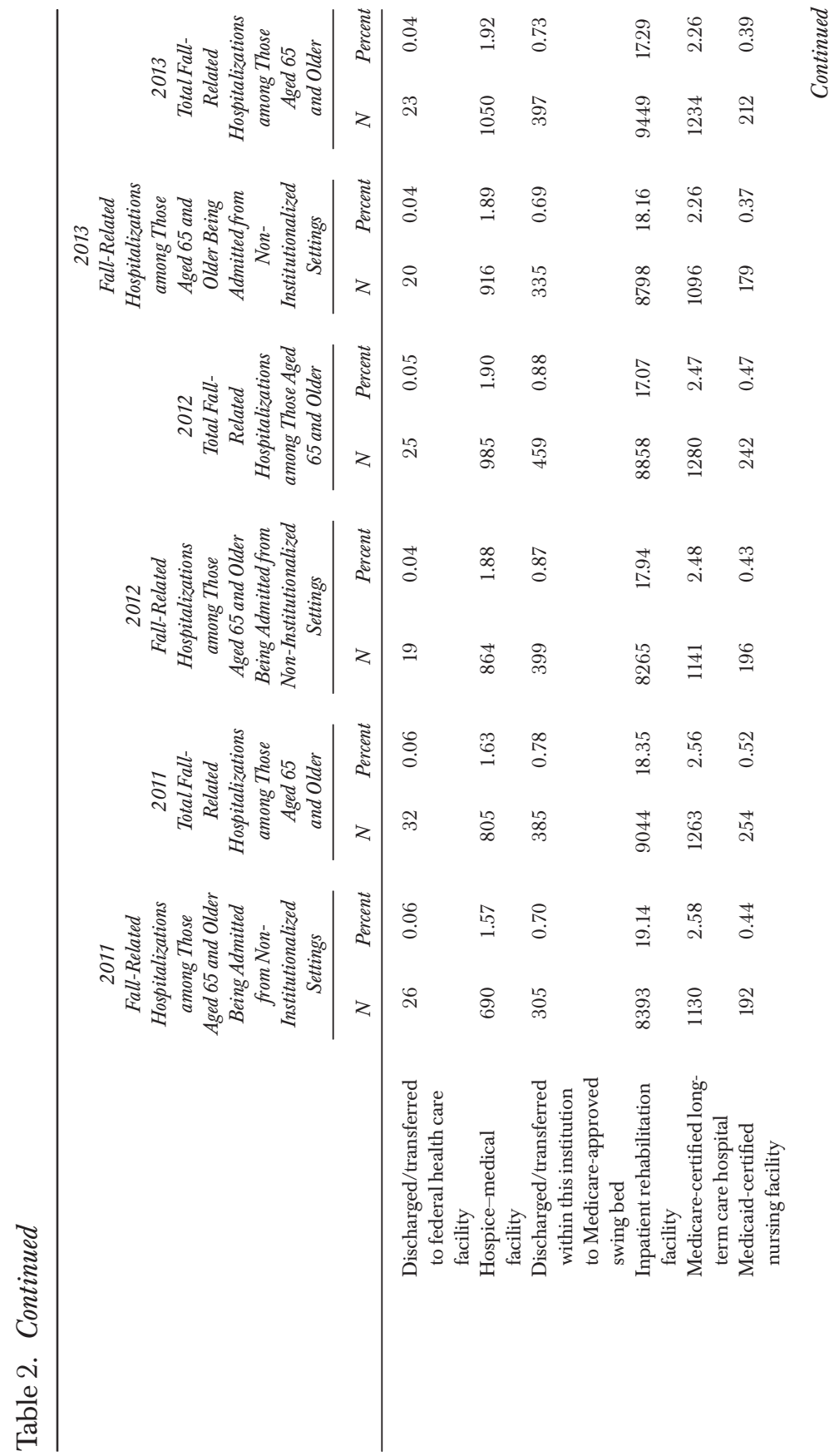




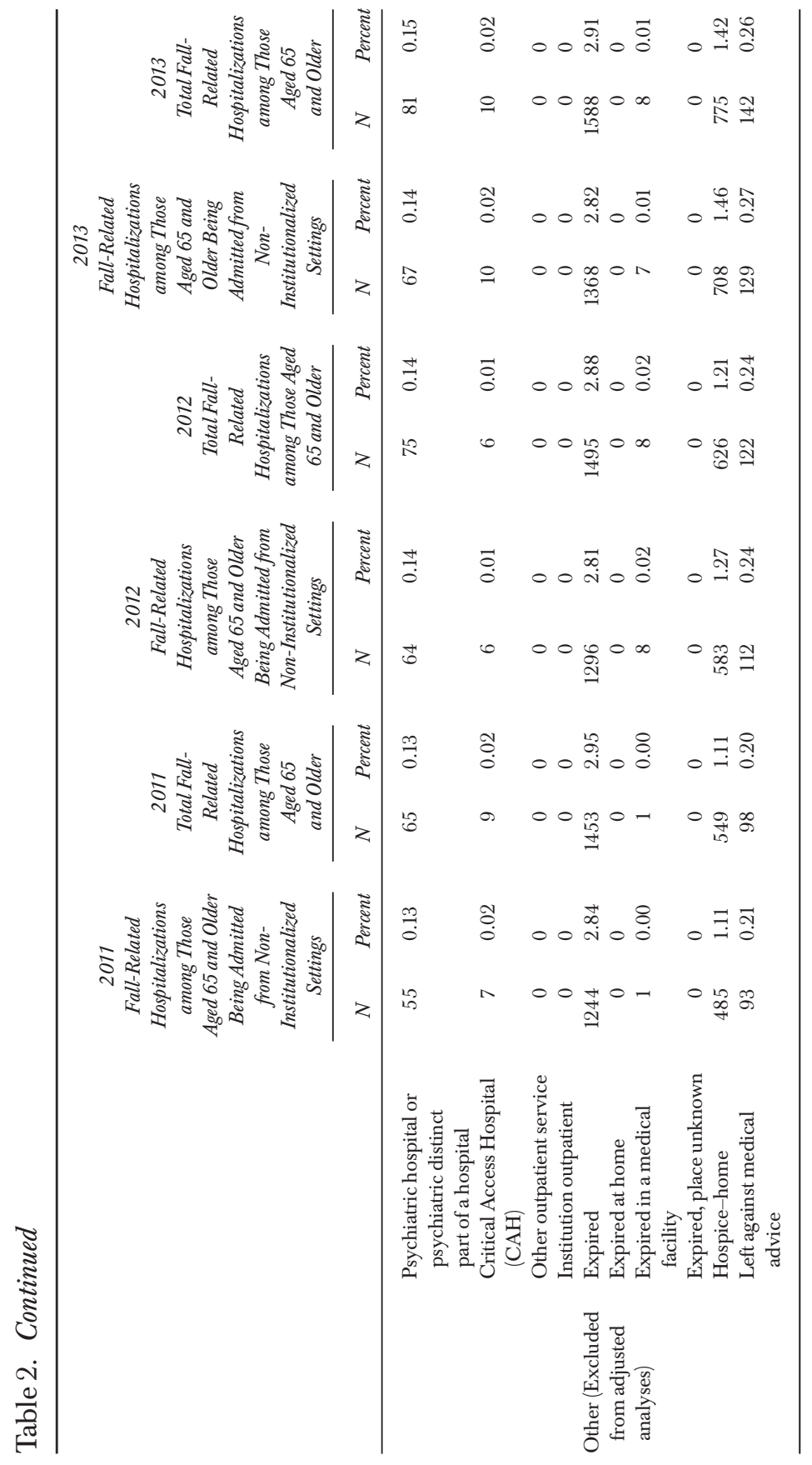


adjusting for all other terms in the model. In contrast, factors associated with $(\alpha=0.05)$ a lower likelihood of being discharged to an institutionalized health care setting included the following: being an Asian or Pacific Islander, black, or Hispanic individual versus being a white individual; having the primary source of payment listed as Private versus Medicare; and being admitted from a clinical referral versus a NHCF after controlling for all other terms in the model across all years under study. In 2011, residents of the most metropolitan areas known as large central metropolitan areas were more likely to be discharged to institutionalized settings versus those residing in medium metropolitan or small metropolitan areas, after adjusting for all other terms in the model.

Discharged to HSC Excluding HHC. Table 3 presents results for the likelihood of being discharged to an institutional health care setting versus HSC. Aside from the results of comparisons across race, results of the model with $H S C$ (Table 3) were similar to that of the model using a combined variable for discharge status of $H S C$ or $H H C$ (Table 3). The lower likelihood of being discharged to an institutionalized setting in the previous set of models was not consistent across all years of study for Asian or Pacific Islander individuals when compared to white individuals (no difference detected in 2011) or for black individuals when compared to white individuals (no difference in 2013; Table 3).

Discharged to HHC Excluding HSC. Table 3 presents results for the adjusted analyses for the likelihood of being discharged to an institutional health care setting versus HHC. Aside for comparisons across sex and teaching status, results of the model with $H H C$ (Table 3) were similar to that of the model using a combined variable for discharge status of $H S C$ or $H H C$ (Table 3). There was no difference detected by sex when modeling the likelihood of discharge to an institutionalized health care setting versus HHC in 2011. When modeling the likelihood of being discharged to an institutionalized health care setting versus $\mathrm{HHC}$, we find no evidence to suggest a difference across teaching status for any year under study. Further, variation between the most metropolitan areas and those areas that were classified as large fringe metropolitan was no longer significant for 2011 after controlling for all other terms in the model.

\section{DISCUSSION}

The number of older adults suffering a fall-related hospitalization who were admitted through NIS/CR prior to admission increased from 2011 


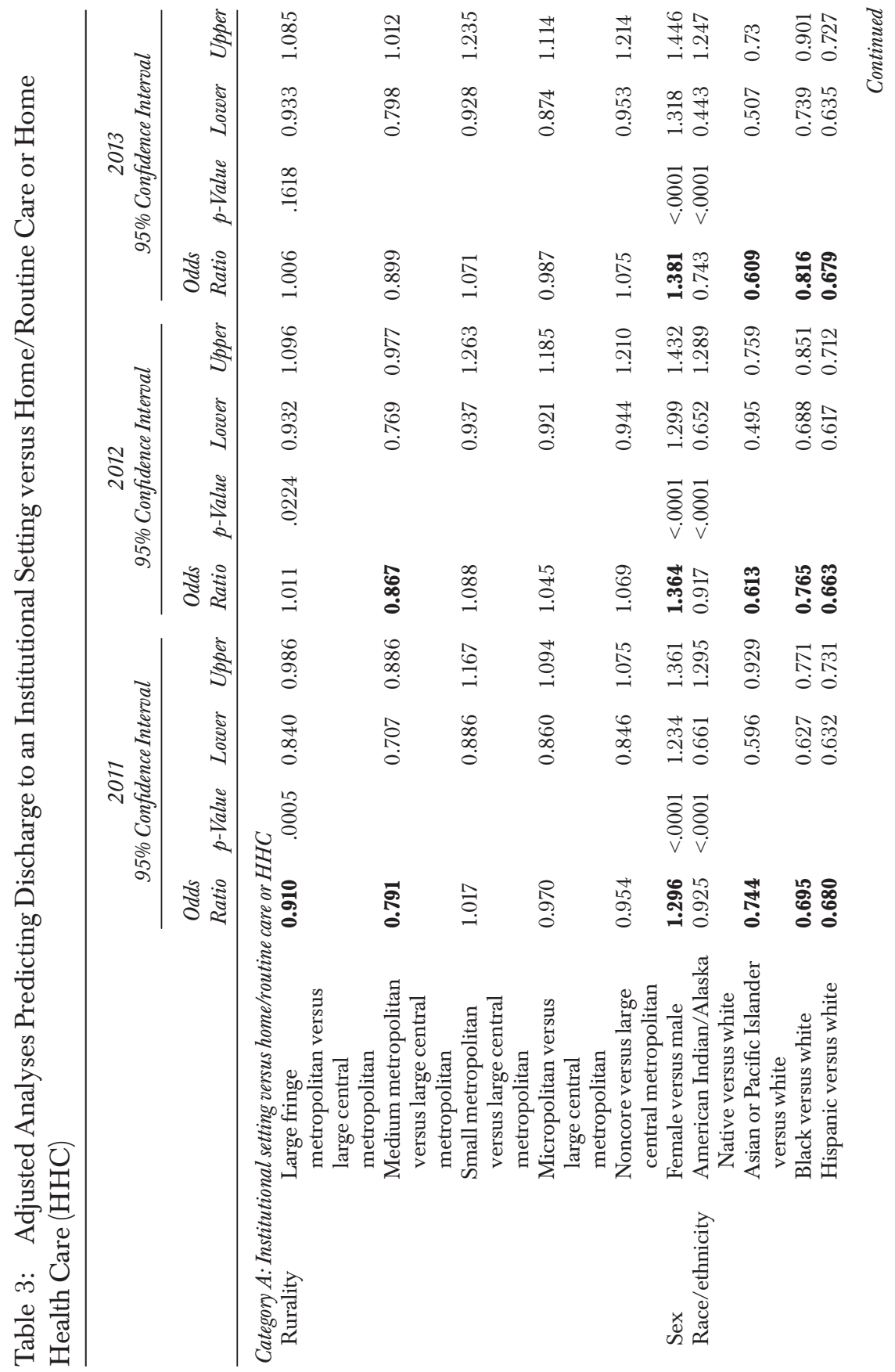




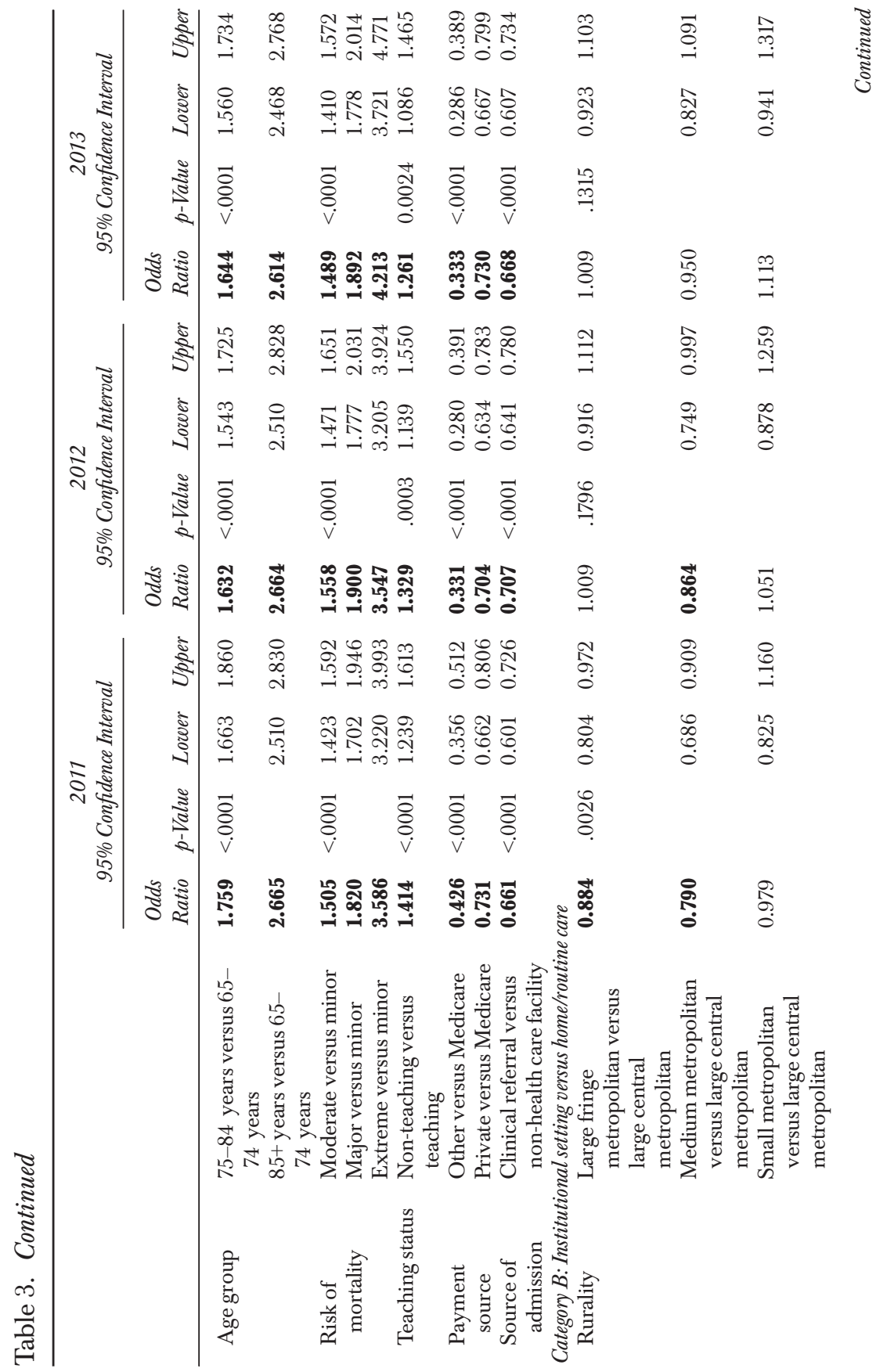




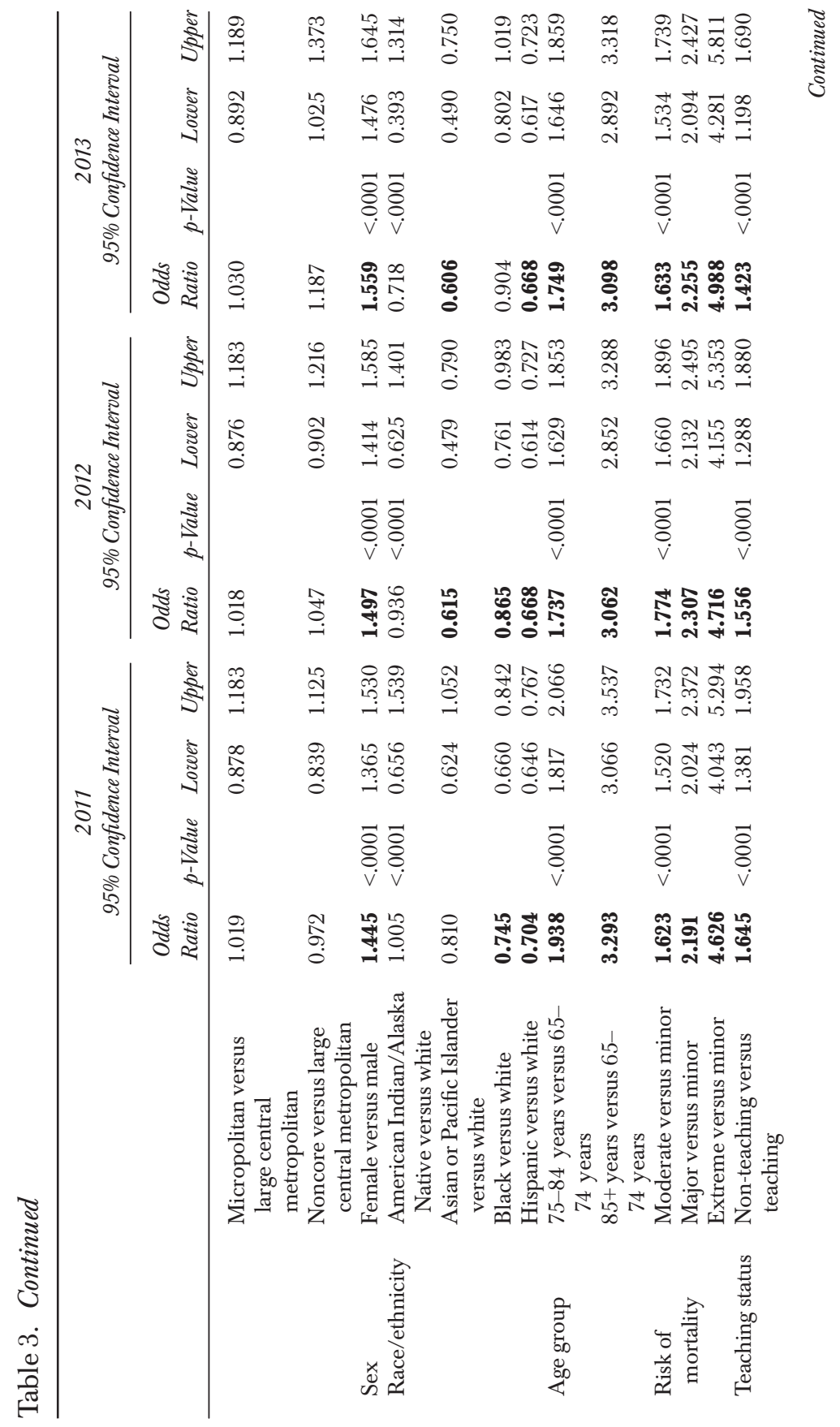




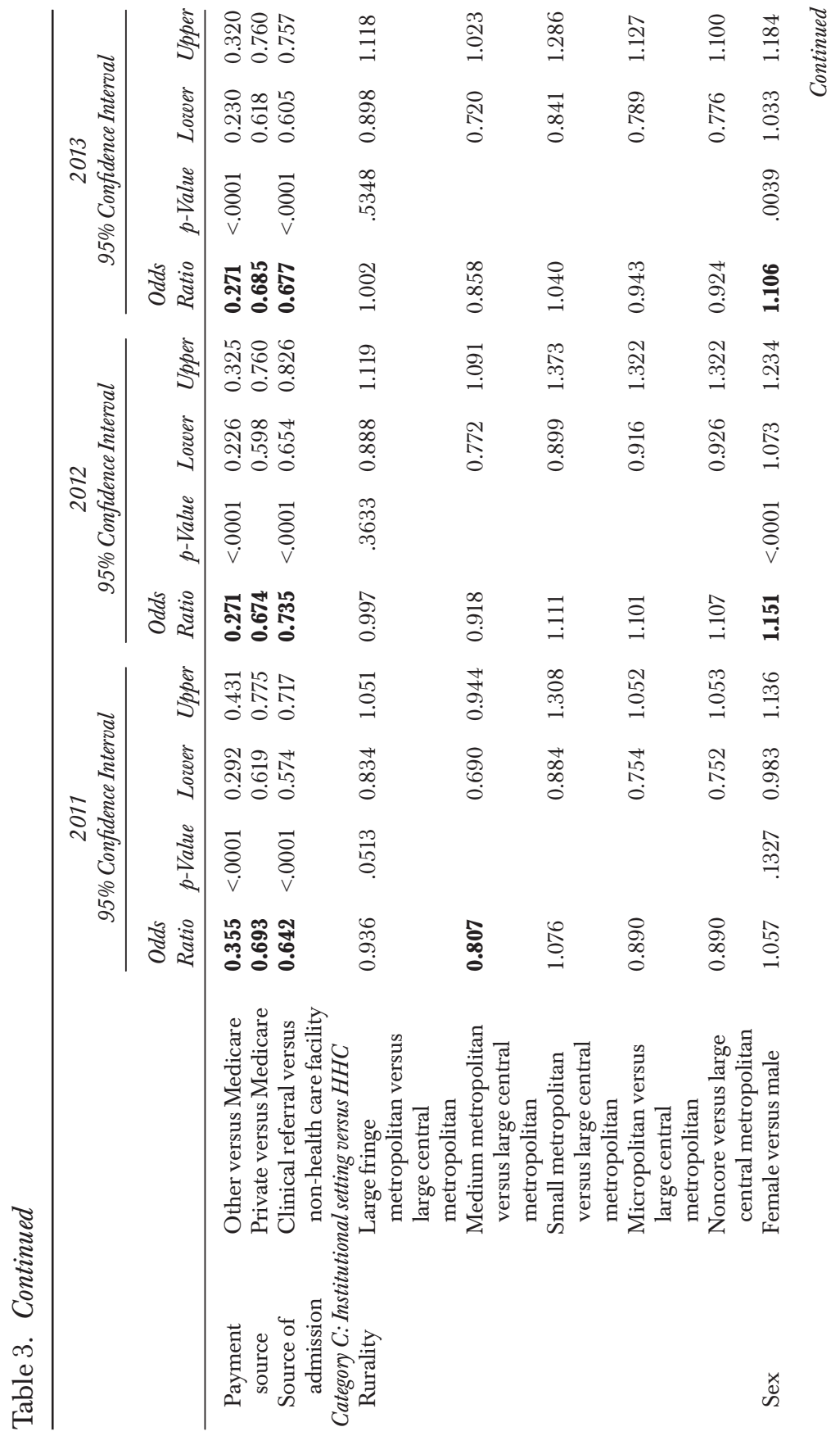




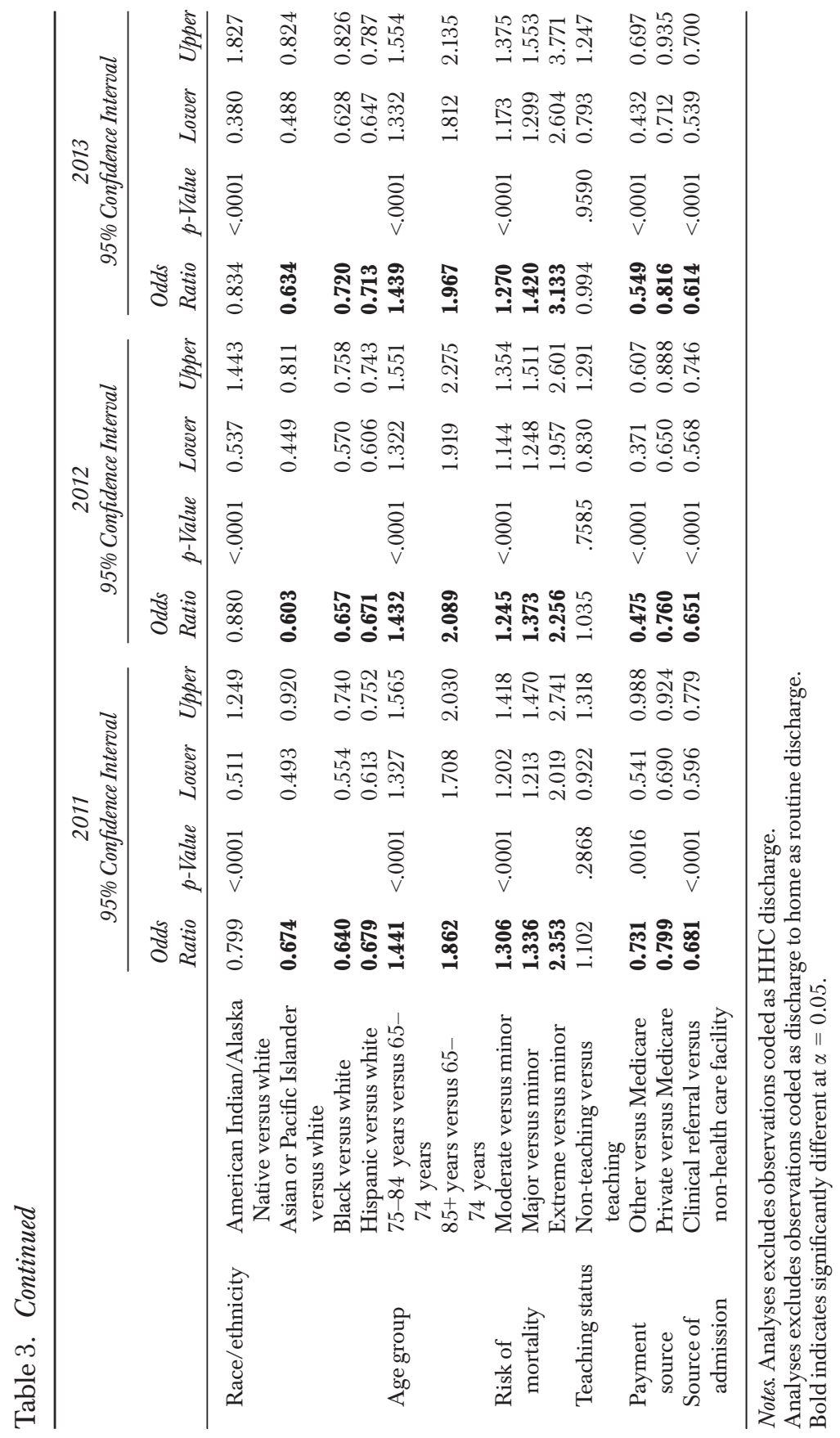


$(n=41,933)$ to $2013(n=46,056)$. This is on par with the rise in the older adult Texas population at approximately 10 percent (TDADS 2014), mirroring the growth in fall-related discharges at approximately 10 percent for the same timeline (2011-2013). However, when assessing the number of overall falls (regardless of location prior to admission) among older adults, we find an increase of 11 percent, slightly higher than the growth in the population. The same comparison from 2007 ( $n=42,153$; Smith et al. 2010) to 2013 indicates the percent increase at 29.6 percent, higher than the rate of growth in the Texas population aged 65 and older during the same timeline at 25.9 percent (2007, $n=2,346,996 ; 2013, n=2,954,614)$. Thus, the need to identify potential solutions to this issue is growing in terms of the sheer size of those affected by fall-related hospitalizations.

\section{Limitations}

The scope of this study was representative of a large U.S. state effecting generalizability. The specific outcome associated with fall-related hospitalizations (e.g., hip fracture, cost) or the severity was not identified in the current analyses, but reported elsewhere (Smith et al. 2010; Towne, Ory, and Smith 2014; Towne et al. 2015). Even so, we included risk of mortality to serve as a proxy for severity, which was found to be a strong independent predictor of discharge location/status. In addition, other clinical and patient characteristics (e.g., number of comorbidities) were not available in the data used in this analyses. The inclusion of those with $\mathrm{CR}$ may reflect a variety of possible locations prior to admission. Comparisons of the individual characteristics of all fall-related hospitalizations and all-purpose hospitalizations of those aged 65 and older were not done as the focus of the study was to compare across discharge status not fall-related versus the total hospitalized population. Finally, we were unable to measure the discharge status past immediate discharge.

\section{CONCLUSIONS AND POLICY IMPLICATIONS}

Several differences persisted even after adjusting for sociodemographic characteristics and a measure of severity (risk of mortality). It is likely that both fragmentation of the health delivery system and patients' health care needs contribute to the decision to discharge home or to an institutionalized setting. As more baby boomers reach the earlier ages of older adulthood, more may 
be opting to continue to work and are thereby more likely to be covered on employer-sponsored health insurance. Thus, identifying potential implications of having Medicare serve as the secondary payer with employer-sponsored health insurance serving as the primary payer (Goda, Shoven, and Slavov 2007) for a growing population of older adults is timely given different outcomes (i.e., discharge location) across payers identified.

Given that a prior fall is a major risk factor for a recurrent fall regardless of discharge status (Stalenhoef et al. 2002), identifying where individuals go after suffering a fall can help inform where fall-prevention interventions or fall-prevention intervention referrals may be delivered most effectively. Improvements in discharge planning for older adults suffering fall-related hospitalizations have been suggested as a potential strategy in the prevention of recurrent falls (Lim, Hoffmann, and Brasel 2007).

Discharge planning is a critical part of care with the goal of reducing unexpected readmissions and improving communication within care transitions (Shepperd et al. 2013). The discharge process may include prescription medication management and follow-up medical care (Spinewine et al. 2013) in addition to related self-care planning. The fragmentation of the health care system may make this process more challenging in that pharmacies, primary care settings, post-acute care settings, and hospitals may not exchange information or coordinate service delivery between settings. Thus, the patient is left with the task of communicating information between care settings. This is a task which is not well suited to older adults following a fall-related hospitalization. Evidence suggests that tailored or individualized discharged planning for older adults may be associated with reduced hospital readmissions (Shepperd et al. 2013) and is therefore critical. Policies affecting what is included in standardized discharge planning for this subpopulation should include three essential components: evidence-based fall-prevention programs, communitybased fall-prevention programs, and information on how to access those programs.

\section{ACKNOWLEDGMENTS}

Joint Acknowledgment/Disclosure Statement: We would like to acknowledge these affiliated institutions: Texas A\&M University; the University of Georgia; the University of Memphis; University of Texas Southwestern Medical Center; and the University of Arkansas for Medical Sciences as listed on our respective affiliations. 
All authors declare no relevant financial interests, activities, relationships, and affiliations (other than those affiliations listed in the title page of the manuscript) including, but not limited to, employment, affiliation, funding, and grants received or pending, consultancies, honoraria or payment, speakers' bureaus, stock ownership or options, expert testimony, royalties, donation of medical equipment, or patents planned, pending, or issued.

Disclosures: None.

Declaimer: None.

\section{REFERENCES}

Bell, B. A., M. Ene, and J. Schoeneberger. 2013. "A Multilevel Model Primer Using SAS PROC MIXED." SAS Global Forum: Statistics and Data Analysis. Conference Proceedings, Paper 433-2013, pp. 0-19.

Das, A., E. C. Norton, D. C. Miller, and L. M. Chen. 2016. "Spending in the Post-Acute Care Setting Accounts for a Sizeable Portion of Spending Associated with a Hospitalization Episode.” JAMA Internal Medicine 176 (1): 117.

Goda, G. S., J. Shoven, and S. N. Slavov. 2007. "A Tax on Work for the Elderly: Medicare as a Secondary Payer." National Bureau of Economic Research. NBER Working Paper No. 13383, pp. 1-25.

Lim, H. J., R. Hoffmann, and K. Brasel. 2007. "Factors Influencing Discharge Location after Hospitalization Resulting from a Traumatic Fall among Older Persons." Journal of Trauma and Acute Care Surgery 63 (4): 902-7.

Masud, T., and R. O. Morris. 2001. "Epidemiology, of Falls." Age and Ageing 30: 3-7.

Mor, V., M. Rahman, and J. McHugh. 2016. "Accountability of Hospitals for Medicare Beneficiaries' Postacute Care Discharge Disposition.” JAMA Internal Medicine 176 (1): 119 .

Rice, N., and A. Leyland. 1996. "Multilevel Models: Applications to Health Data." Journal of Health Services Research 1 (3): 154-64.

Shepperd, S., N. A. Lannin, L. M. Clemson, A. McCluskey, I. D. Cameron, and S. L. Barras. 2013. "Discharge Planning from Hospital to Home." Cochrane Database of Systematic Reviewes 1 (1).

Smith, M., M. Ory, C. Beasley, K. Johnson, M. Wernicke, and R. Parrish. 2010. "Falls among Older Adults in Texas: Profile from 2007 Hospital Discharge Data." TPHA Journal 62 (1): 15-20.

Spinewine, A., C. Claeys, V. Foulon, and P. Chevalier. 2013. "Approaches for Improving Continuity of Care in Medication Management: A Systematic Review." International Journal for Quality in Health Care 25 (4): 403-17.

Stalenhoef, P., J. Diederiks, J. Knottnerus, A. Kester, and H. Crebolder. 2002. “A Risk Model for the Prediction of Recurrent Falls in Community-Dwelling Elderly: A Prospective Cohort Study." Journal of Clinical Epidemiology 55 (11): 1088-94. 
Sterling, D. A., J. A. O’Connor, and J. Bonadies. 2001. "Geriatric Falls: Injury Severity Is High and Disproportionate to Mechanism." Journal of Trauma and Acute Care Surgery 50 (1): 116-9.

TDADS. 2014. "Texas Department of State Health Services Census Data“ [accessed on June 06, 2014]. Available at http://www.dshs.state.tx.us/chs/popdat/detailX. shtm

THHS. 2011. Texas Hospital Inpatient Discharge Public Use Data File. Austin, TX: Texas Health and Human Services, Center for Health Statistics.

Tinetti, M. E., and C. S. Williams. 1997. "Falls, Injuries Due to Falls, and the Risk of Admission to a Nursing Home." New England Journal of Medicine 337 (18): 127984 .

Towne, S., M. Ory, and M. Smith. 2014. "Cost of Fall-Related Hospitalizations among Older Adults: Environmental Comparisons from the 2011 Texas Hospital Inpatient Discharge Data.” Population Health Management 17 (6): 351-6.

Towne, S. D., M. L. Smith, A. Yoshikawa, and M. G. Ory. 2015. "Geospatial Distribution of Fall-Related Hospitalization Incidence in Texas." Journal of Safety Research 53: $11-6$.

\section{SUPPORTING INFORMATION}

Additional supporting information may be found online in the supporting information tab for this article:

Appendix SA1: Author Matrix. 